\title{
Les évangélistes en Amérique Latine : De l'expression religieuse à la mobilisation sociale et politique transnationale
}

\section{Ariel Colonomos}

\section{(2) OpenEdition \\ Journals}

Édition électronique

URL : http://journals.openedition.org/conflits/201

DOI : $10.4000 /$ conflits.201

ISSN : 1777-5345

Éditeur :

CCLS - Centre d'études sur les conflits lilberté et sécurité, L'Harmattan

Édition imprimée

Date de publication : 15 octobre 1994

ISSN : 1157-996X

Référence électronique

Ariel Colonomos, «Les évangélistes en Amérique Latine : De l'expression religieuse à la mobilisation sociale et politique transnationale », Cultures \& Conflits [En ligne], 15-16 | automne-hiver 1994, mis en ligne le 15 mars 2006, consulté le 30 mars 2021. URL : http://journals.openedition.org/conflits/201 ; DOI : https://doi.org/10.4000/conflits.201

Ce document a été généré automatiquement le 30 mars 2021

Creative Commons License 


\title{
Les évangélistes en Amérique Latine : De l'expression religieuse à la mobilisation sociale et politique transnationale
}

\author{
Ariel Colonomos
}

Le développement du protestantisme à travers ses nouvelles composantes constitue un des traits saillants de l'évolution récente du paysage socio-culturel latino-américain. Dans des pays d'Amérique centrale comme le Mexique, le Guatemala, ou le Nicaragua, ou bien en Amérique andine en Colombie, au Pérou et en Bolivie, ainsi qu'au Chili ou bien en Argentine, le pentecôtisme ${ }^{1}$, un des mouvements religieux dans la lignée du méthodisme ${ }^{2}$, semble en plein essor. Cette dynamique religieuse désignée par le terme évangélisme ${ }^{3}$ concerne en tout premier lieu le pentecôtisme et ses marges en amont et en aval, mais n'intègre pas d'autres courants issus de la matrice protestante originelle comme les Adventistes du septième jour, les Mormons ou les Témoins de Jéhovah4 L'émergence de cultes pentecôtistes et néo-pentecôtistes participe de l'avènement de formes de religiosité particularistes au sein desquelles l'individu renouvelle son identité et son expression sociales. En effet, la logique appliquée de la dynamique évangéliste repose sur une destruction créatrice, la fondation d'un nouveau groupe à partir de l'ancien. De nouveaux enjeux se dessinent sur une trame culturelle qui intègre les mutations récentes du monde contemporain, dans la perspective de profonds changements sociaux et politiques. Des forces politiques et institutionnelles, comme l'Etat et l'Eglise catholique, sont questionnées dans leur action. Cette tendance est corrélative d'une dynamique au sein de laquelle des sous-groupes infra-nationaux, nationaux et transnationaux sont confrontés à l'Etat et aux institutions, en tant qu'interlocuteurs et partenaires du changement social. Cette idée se trouve à l'oeuvre dans les relations internationales d'aujourd'hui à l'image d'un monde où s'expriment avec de plus en plus de vigueur les particularismes ethniques, religieux, culturels ou identitaires ${ }^{5}$. Plusieurs logiques transnationales sont à l'oeuvre dans l'émergence de ces mouvements religieux en Amérique latine. L'étude de ces différentes logiques permet 
une meilleure compréhension du comportement de ces acteurs à l'échelle de chaque société. L'opérationnalisation de ces logiques témoigne de la faculté des acteurs sociaux à produire du sens dans des espaces socio-politiques extensibles, à l'échelle d'une région ou d'un continent. Les évangélistes ont désormais acquis une reconnaissance du fait de leur croissance numérique et de leur visibilité au sein des différentes sociétés latino-américaines. Il s'agit d'analyser différentes stratégies liées à leur transnationalité conditionnant l'action politique dans les différentes sociétés internes ${ }^{6}$.

2 La conquête d'espaces sociaux vides La dimension fortement prosélyte de la plupart de ces groupes religieux est à l'origine de l'action transnationale des évangélistes en Amérique latine. Le salut des âmes est le devoir de tout croyant, l'impératif de sa conduite : en sauvant l'individu qu'il s'emploie à convertir il sauve l'humanité. De l'agrégation de ces conduites individuelles dépend l'avènement d'une collectivité et d'un ordre politique édifié sur les bases d'une théologie fondamentaliste. Du point de vue des relations internationales, on peut discerner trois dynamiques complémentaires qui témoignent de l'action sociale des évangélistes dans de nombreux pays latinoaméricains, une dynamique de mission, une dynamique de croisade et une dynamique diplomatique. La dynamique de mission fait partie de l'héritage légué par les différentes vagues missionnaires, tout d'abord les protestants venus d'Europe à la fin du XIXe siècle, puis, plus tard, les évangélistes des Etats-Unis. Cet impératif de mission a été intégré par les Eglises pentecôtistes qui planifient leur action en fonction d'objectifs quantitatifs d'évangélisation. Les évangélistes se concentrent tout particulièrement sur des groupes cibles (unreached groups) définis suivant des critères, comme l'ethnie, la tribu, la condition sociale. Des centres de recherche et d'information établissent à l'échelle mondiale les listes de ces différents groupes. Cette méthodologie témoigne du prosélytisme virulent de ces religieux qui emploient certaines ressources de la transnationalité afin de mener à bien leur projet de réforme de la société. Les groupes évangélistes ont largement recours aux techniques audiovisuelles et informatiques, et opèrent bien souvent en coopération avec des entreprises de communication audio ou vidéo, responsables des opérations de médiatisation indispensables à tout projet d'évangélisation massive. La logique religieuse s'appuie ici sur une logique économique d'entreprise transnationale dont la structure même est le réseau: d'une part un centre qui fournit avant tout la logistique, l'infrastructure nécessaire à la reproduction du mouvement et les ressources économiques nécessaires afin de mener à bien les premiers projets d'investissement, d'autre part des satellites qui emploient une main d'oeuvre autochtone sensibilisée à l'utilisation de techniques importées et résolue à s'autonomiser du centre. Si bien souvent les Eglises évangéliques en Amérique latine emploient des pasteurs autochtones et sont donc considérées, à ce titre, comme des Eglises nationales, les organisations missionnaires et les réseaux médias sont, quant à eux, pour la plupart d'origine étrangère. Dès lors, le critère de la nationalité ne fait plus sens pour définir des Eglises et des organisations dans un univers où la collecte des ressources, l'expression des valeurs et la formation des dirigeants s'organisent à l'échelle mondiale à travers des réseaux inter-individuels. Cette dynamique de mission ne saurait être effective en Amérique latine sans une deuxième composante de la transnationalité évangéliste : la dynamique de croisade. La mission et la planification des conversions sont organisées dans des contextes où la dynamique d'entreprise est confortée par une dynamique militaire. L'Eglise est une organisation qui motive ses fidèles autour de la définition de projets à vocation transnationale: collecte de fonds, envoi de missionnaires et de cadres, édification de 
réseaux de communication et de mobilisation, consolidation de ces réseaux. La conjonction de ces deux dynamiques se rencontre dans un mouvement historique auquel s'identifient largement de nombreux groupes évangélistes en Amérique latine : la croisade. C'est le terme employé par les acteurs eux-mêmes pour désigner des opérations de médiatisation, de conversions massives et de formation des dirigeants dans des pays comme le Guatemala, le Venezuela, la Colombie, le Chili ou l'Argentine. Ces croisades sont menées par des pasteurs dont le charisme, la réputation mondiale dont ils jouissent dans l'univers évangéliste et les moyens économiques dont ils disposent, leur permettent de créer des événements fortement spectaculaires afin de mobiliser les masses dans les grands centres urbains latino-américains ${ }^{7}$. Ces événements sont d'une importance considérable pour les communautés évangélistes à l'échelle de chaque société. Ils sont l'occasion pour les dirigeants locaux de conforter leur pouvoir grâce à la légitimation symbolique et pratique que fournit la présence d'un éminent pasteur étranger. Les pasteurs des différentes Eglises sont rassemblés dans cette mobilisation qui fixe des objectifs de travail à moyen terme. Enfin, ces rencontres sont bien souvent l'occasion pour les évangélistes de se positionner face à l'Etat et à l'Eglise catholique. L'évangéliste transnational apporte des ressources destinées à courtiser les bureaucraties latino-américaines. Dans des situations de pénurie de ressources, comme ce fut le cas par exemple au Guatemala en 1976 après le tremblement de terre ${ }^{8}$, les évangélistes présentent au pouvoir politique un de leurs représentants chargé d'assurer une fonction d'intermédiation auprès de l'Etat et des gouvernants. La croisade est également l'occasion de se mobiliser contre l'Eglise catholique en lui opposant, sur la scène de l'espace public, une autre diplomatie transnationale. La présence au Chili en 1987 du télé-évangéliste nord-américain Jimmy Swaggart, une année avant la venue du Pape, témoigne de la volonté expresse des dirigeants évangélistes de remettre en cause le monopole de la représentation religieuse de l'Eglise catholique, à un moment où la relation Eglise-Etat était marquée par une forte tension du fait de l'opposition de certains évêques au régime militaire du général Pinochet. Aussi, la logique transnationale évangéliste comprend également une dynamique diplomatique. L'absence de centre implique des configurations particulières: une difficile construction institutionnelle, la coalescence ponctuelle autour de la venue d'un dirigeant étranger, l'émergence et la multiplication de nouveaux acteurs. Néanmoins, malgré l'absence de hiérarchie bureaucratique ${ }^{9}$, des ambassades existent dans la plupart des villes latino-américaines. La fonction de ces bureaux consiste tout d'abord à assurer une intermédiation auprès des gouvernants : canaliser des ressources, en provenance principalement des Etats-Unis, mobiliser les organisations locales et veiller à ce que le travail missionnaire ne soit pas remis en cause par les autorités de l'Etat. Des espaces de représentation sont convoités par ces groupes religieux désireux d'accéder à une légitimité sociale dans les sociétés internes. Grâce aux ressources transnationales, symboliques, logistiques et économiques, les ambassades sont chargées de favoriser ce passage à l'action dans le cadre de l'Etatnation. Des réseaux de diplomatie informelle sont tenus de veiller aux bons rapports avec l'Etat afin de gagner du terrain sur l'Eglise catholique. Dans l'analyse de ces mouvements, la perspective diachronique nous engage à dresser un constat: la transnationalité est un des recours majeurs que les évangélistes ont utilisé depuis deux décennies pour conquérir des espaces sociaux vides. Les Eglises protestantes sont pourtant présentes en Amérique latine depuis le début du siècle ${ }^{10}$. Ainsi, dès 1882 , le chef de l'Etat guatémaltèque avait invité la première mission nord-américaine à 
s'établir dans le pays. Cependant, leur prise de parole dans l'espace public en tant que mouvements évangéliques, et la conquête d'espace sociaux dans cette arène est un phénomène récent. Cette innovation est susceptible d'être analysée en tenant compte de l'effectivité des mobilisations transnationales qui sous-tendent, sur les différentes scènes stato-nationales, l'émergence de ces nouveaux acteurs.

Quelles transnationalités? La transnationalité évangéliste est tributaire d'une dynamique de relations inter-individuelles et inter-organisationnelles structurée autour de multiples rencontres, de conférences et de colloques organisés à l'échelle régionale, nationale et mondiale. Comme nous l'avons souligné précédemment, les principaux acteurs de ces réunions sont des pasteurs dont l'importance est reconnue suivant l'appréciation de la taille de leur Eglise et de l'influence de celle-ci au sein de la société. La transnationalité, dans le cadre de la diffusion des évangélistes dans les sociétés latino-américaines, a pour fondement la reproduction d'un modèle que les groupes exportateurs ont pour vocation de proposer. Ainsi, se créent des espaces et des réseaux transnationaux construits autour d'un même modèle et d'une même dynamique. Les télé-évangélistes sont un exemple frappant de cette logique qui favorise une multiplication des acteurs à travers la transnationalité. Les téléévangélistes, les pasteurs ayant accès aux médias et aux ressources économiques que ces médias permettent de collecter, étaient jusqu'à la fin des années 70 principalement nord-américains. Désormais, Billy Graham, Jimmy Swaggart ou Pat Robertson n'ont plus le monopole de la représentation religieuse évangéliste sur le sous-continent latino-américain. En l'espace de deux décennies, s'est créée une diplomatie religieuse autochtone, habituée aux rouages politiques et sociaux des bureaucraties locales. Cette diplomatie a été formée aux Etats-Unis et possède ses propres infrastructures dont le siège, bien souvent, se trouve dans ce pays du fait des conditions économiques et fiscales particulièrement favorables ${ }^{11}$. C'est par exemple le cas de deux célèbres évangélistes argentins, Luis Palau et Alberto Mottesi. Ces deux personnages sont tout particulièrement représentatifs $d u$ recours à la transnationalité dans l'univers évangéliste. Après avoir travaillé en Amérique latine ${ }^{12}$ et acquis une reconnaissance dans cet espace régional, ils se sont désormais établis aux Etats-Unis en fondant leur propre entreprise religieuse. Leur accès aux ressources est facilité par leurs nombreux contacts auprès des groupes évangélistes nord-américains et auprès de l'importante communauté hispanique résidant dans les grands centres urbains des Etats-Unis. A la croisée de plusieurs cultures, ils incarnent des figures prophétiques, dont la fonction d'intermédiation avec l'Etat est une ressource précieuse, et utilisée par les différentes communautés évangélistes latino-américaines dans leur stratégie d'occupation de l'espace public. Les dirigeants évangélistes ont pour hantise la reproduction de leurs structures et la croissance de leur population. Aussi l'intégration de dirigeants locaux dans les instances de décision, de représentation et d'intermédiation auprès de l'Etat est rapidement devenue un impératif dans l'organisation des ressources humaines de ces entreprises transnationales. Le réseau évangéliste s'est progressivement construit autour de plusieurs principes destinés à favoriser son extension: économie des structures, primat de l'individu sur l'organisation, rentabilité économique de l'entreprise religieuse, valorisation de la transnationalisation. A l'échelle continentale, cette logique de reproduction correspond à un projet qui motive les acteurs dans la construction de leurs relations au sein du réseau. En suivant un modèle, ces acteurs religieux consolident un réseau bâti autour de la présence d'individus charismatiques, véritables guides dans la mobilisation politique. La théologie évangéliste, misant 
notamment sur les dons de la prophétie, incite l'individu à créer une organisation au sein de laquelle il régnera en maître, délégué par Dieu, jusqu'au moment où un rival s'écartera du groupe pour fonder sa propre entreprise religieuse en provoquant, de fait, une scission. Une logique sociale rend possible l'énonciation de ce projet: la logique d'imitation. Le pasteur se fait l'écho de la puissance de Dieu sur terre. Aussi, le spectaculaire auquel le recours à la transnationalité donne lieu est une manifestation de cette imitation de Dieu qui mobilise les masses en Amérique latine. En philosophie, puis en sociologie, l'étude des religions a déjà permis d'explorer la portée de ce phénomène, l'imitation de Dieu et le pouvoir politique des fonctionnaires religieux, et de constater dans quelle mesure il guide bon nombre de conduites dans des espaces culturels aussi différents que l'islam, le catholicisme ou le protestantisme. Du point de vue des relations internationales, l'exemple des évangélistes en Amérique latine met en lumière une logique d'imitation opérationnalisée à travers un réseau religieux transnational. La logique d'imitation implique l'incorporation de nouveaux acteurs, des évangélistes ou des télé-évangélistes accomplissant de nombreuses fonctions sociales et politiques, prophétie, autorité charismatique, intermédiation avec les gouvernants. L'imitation est au fondement de la multiplication des acteurs et des espaces sociaux investis par les évangélistes. C'est aussi en accord avec cette conception de l'action sociale, à savoir l'imitation, que sont organisées les multiples réunions entre les différents dirigeants à l'échelle continentale et mondiale: se multiplier, former de nouveaux cadres pour transformer la société. Les évangélistes ont construit leur propre rôle d'intermédiaire: envoyés de Dieu auprès des masses et intermédiaires entre les communautés religieuses et l'Etat. Ce rôle d'intermédiation intègre deux fonctions proprement politiques: l'éducation des masses à travers l'opérationnalisation d'un modèle et la réforme du social que permet la canalisation des ressources. Des chaînes de solidarité se créent dans des espaces de mobilisation extensibles. Des processus de clientélisation transnationaux s'organisent autour de ces personnages clés, les évangélistes, des hommes providentiels dont le charisme permet de guider cette économie de l'offre et du don ${ }^{13}$. Bien souvent, les réunions nationales ne sont que des répliques de ces grands forums continentaux et mondiaux auxquels participent les dirigeants les plus importants ${ }^{14}$. Aussi, ces rencontres ont pour fonction d'entériner le pouvoir des anciens et de sélectionner les futurs dirigeants. La logique de l'imitation guide les conduites des acteurs au cours de ces réunions : ordonné par Dieu, le pasteur tire sa légitimité de la prophétie qui lui a permis d'accéder au statut d'élu. Nombre de grandes figures de l'univers évangéliste latino-américain ont utilisé leur expérience de la transnationalité afin d'accéder à des charges de pouvoir dans des communautés religieuses locales. Aussi, certains pasteurs latino-américains ont été ordonnés aux Etats-Unis. Le réseau permet l'émergence des successeurs dans un univers où la concurrence est la conséquence d'une dynamique de reproduction, sans qu'aucune instance hiérarchique centralisée n'ait le pouvoir de fixer définitivement les règles bureaucratiques de fonctionnement dans la désignation des élus. La transnationalité évangéliste en Amérique latine possède une caractéristique qui va au-delà des logiques de reproduction et d'imitation: l'émulation. Force est de constater la puissance de mobilisation de certaines Eglises dans des pays comme le Guatemala, où une nouvelle génération de pasteurs pentecôtistes a imposé un programme mettant en cause le refus de s'immiscer dans les affaires publiques et dans l'activité politique partisane ou gouvernementale, qui caractérisait la tradition pentecôtiste latino-américaine jusqu'à la fin des années 70. Ce néo-pentecôtisme concerne une partie de la population, les 
classes moyennes et certains dirigeants, jusqu'alors rétive aux appels des pasteurs . Plusieurs Eglises témoignent au Guatemala de cette conception nouvelle de l'activité religieuse. A l'image des deux congrégations des deux présidents évangélistes qu'a connu le pays en l'espace d'une décennie, le général Rios Montt membre de l'Eglise du Verbe et président de 1982 à 1983, et l'ingénieur Jorge Serrano membre de l'Eglise Shaddaï, président de 1991 à 1993. A des degrés divers, les dirigeants de ces Eglises ont bénéficié de l'apport de flux transnationaux pour fonder des organisations en rupture avec la tradition religieuse évangéliste locale qui résultait elle-même d'une précédente importation. Deux mobilisations se sont alors rencontrées à travers la transnationalité : le néo-pentecôtisme latino-américain et des organisations qui émergent aux Etats-Unis à partir de la fin des années 70. En Amérique latine, nombre d'Eglises néo-pentecôtistes sont liées aux organisations nord-américaines qui prônent un engagement dans la politique, en tant que chrétiens évangélistes, et une moralisation de la vie publique ${ }^{15}$. Des liens transnationaux, à travers l'interconnaissance des dirigeants, témoignent des deux logiques déjà mentionnées, reproduction et imitation, mais aussi, et c'est parfois la composante essentielle, logique d'émulation. Les dirigeants de la nouvelle droite religieuse américaine sont des modèles que les pasteurs latino-américains cherchent à dépasser. L'ouverture de certains espaces sociaux permet ce dépassement, comme en témoigne l'accès aux postes de direction de l'Etat dans le cas de Rios Montt et de Serrano au Guatemala. Là où les évangélistes nord-américains ne peuvent qu'échouer, dans la conquête du pouvoir exécutif par exemple, les évangélistes latino-américains bénéficient d'avantages compétitifs et d'opportunités politiques décisives. Le Guatemala est le pays où ces phénomènes de mobilisation transnationale ont connu un succès d'une ampleur considérable. Cependant, ces mobilisations identitaires concernent d'autres pays du continent latino-américain. Des Eglises néo-pentecôtistes existent dans des pays comme le Venezuela et le Chili. Elles sont organisées suivant les mêmes modèles: des groupes autonomes et prospères soudés par l'autorité charismatique des dirigeants à la recherche d'un pouvoir politique dans la direction des affaires de l'Etat. Les Eglises néo-pentecôtistes fondent leurs propres réseaux nationaux et transnationaux, conscientes des différences de statut et d'objectifs qui marquent leur séparation avec un univers et un modèle piétistes, un "refuge des masses " ${ }^{16}$ que la nouvelle génération de dirigeants réfute. On assiste ainsi à une logique d'émulation qui apporte un démenti à la thèse de la dépendance souvent invoquée pour identifier ces phénomènes de transnationalisation. Les importations de ressources économiques, religieuses, logistiques et politiques des Etats-Unis, où sont établis de nombreux centres de ces mouvements, ne doivent pas occulter la logique proprement transnationale de ces groupes. En effet, de nombreuses associations latino-américaines ont vu le jour et participent activement à la mobilisation des évangélistes dans la plupart des pays du sous-continent ${ }^{17}$. L'invention théologique rejoint ainsi la dynamique transnationale de diffusion et de mobilisation. Le réseau est le cadre qui rend possible cette mobilisation transnationale. Par ailleurs, de nombreuses Eglises latino-américaines se sont installées aux Etats-Unis dans les grands centres urbains comme Los Angeles, New York ou Miami. Ces décisions sont motivées par la volonté de prendre en charge les migrants latino-américains en poursuivant les objectifs d'une diplomatie par le bas : la théologie politique de l'alliance ${ }^{18}$ est à l'origine de ce contournement des Etats et de l'intérêt prononcé des évangélistes pour les groupes dans la vie politique transnationale. Par là même, les dirigeants évangélistes latino-américains peuvent rester en contact avec un univers nord-américain producteur d'innovations. 
4 La question «sectaire » et la construction transnationale « d'une Eglise » évangélique De nombreux auteurs, principalement des sociologues de la religion, ainsi que certains porte-parole de l'Eglise catholique, tendent à désigner les évangélistes en utilisant le terme de « secte ». Force est de constater bien souvent, c'est particulièrement net en ce qui concerne l'Eglise catholique, le dessein de discréditer la légitimité sociale et religieuse de ces mouvements. Du point de vue des relations internationales, un phénomène tout particulièrement intéressant est à l'oeuvre actuellement en Amérique latine : le passage de la secte à l'Eglise ${ }^{19}$. Cette transition a pour toile de fond et pour enjeu la transnationalité et les relations entre les différents acteurs. Les flux transnationaux permettent la structuration d'une diplomatie qui a pour dynamique la multiplication des intervenants sur une scène riche en innovations. Ces acteurs construisent leur légitimité en reliant localisme, leur travail dans les sociétés latinoaméricaines, et mondialisme, le recours aux instances légitimantes d'un ordre religieux en perpétuel renouvellement. Ainsi, des processus d'institutionnalisation sont à l'oeuvre. On préférera dès lors utiliser le terme d'Eglises indépendantes pour désigner la place de ces acteurs dans les relations internationales, position intermédiaire entre la secte et l'Eglise ${ }^{20}$. La dynamique associative et transnationale de ces groupes, qui intègrent aussi bien des associations de missionnaires que des clubs pour élites économiques et politiques, atteste de la présence d'un objet dont la vocation serait une mobilisation transnationale à l'image d'une Eglise unifiée. Ce projet se heurte néanmoins à la volonté d'indépendance des différents dirigeants. Une lecture de ces groupes en terme de réseaux s'impose, des réseaux dont il faut, à l'aide de l'étude des liens transnationaux à l'intérieur desquels ils s'inscrivent, recomposer la logique qui a conduit aux mobilisations politiques. Le passage d'une dynamique de secte à une dynamique de type Eglise est corrélatif de ces mobilisations. Les centres de diffusion ne sauraient contrôler les groupes qu'ils ont permis de constituer. Cette incapacité de contrôle hypothèque radicalement toute possibilité de réussite dans un processus d'institutionnalisation.

5 Logique d'institution et logique de réseau Une confusion règne en Amérique latine dans la distinction entre protestantisme et évangélisme. D'un point de vue politique, il est possible de reconnaître les évangélistes en Amérique latine au type de mobilisation transnationale qu'ils organisent. Dans la perspective d'une étude en relations internationales, deux comportements antagonistes témoignent des positions des Eglises protestantes et des Eglises évangéliques face à leur engagement dans la sphère publique et à la définition de leur identité politique. Le protestantisme traditionnel assume par l'intermédiaire de ses Eglises comme certaines Eglises méthodistes ou presbytériennes une position oecuménique. Le Conseil Oecuménique des Eglises, qui siège à Genève, est le référent institutionnel pour ces Eglises protestantes qui s'identifient à une organisation régionale dans la mouvance du COE, le CLAI (Consejo Latino Americano de Iglesias). La logique de l'action de ces Eglises est une logique institutionnelle intégrant en son sein une majorité d'Eglises protestantes dites historiques, qui dépendent bien souvent de leur centre européen ou nord-américain, et une minorité d'Eglises pentecôtistes. Ainsi, l'identité protestante se définit en Amérique latine par sa relation aux institutions oecuméniques. L'espace transnational que ces Eglises occupent est un espace institutionnel marqué par la coopération avec l'Eglise catholique et la critique des injustices sociales. Le rapport aux institutions donne lieu à la mobilisation d'intellectuels, pasteurs et laïcs, désireux de penser le politique à travers le prisme de la théologie protestante. Les répertoires d'action de ces 
organisations font partie intégrante des registres traditionnels de l'opposition politique, comme en témoignent les positions de ces Eglises et de ces ONGs au Guatemala et au Chili : dénonciation de l'oppression, de la pauvreté et de la domination. Force est de constater que ces mouvements sont minoritaires au sein du protestantisme latino-américain, marqué par la présence des Eglises évangéliques radicalement opposées à cette logique sociale et à ce mode d'action de la transnationalité. Une autre organisation régionale s'est constituée en 1982 en même temps que le CLAI et en réaction à l'annonce de sa création: la CONELA (Confraternidad Evangelica Latino Americana). Il s'agit là d'un réseau d'évangélistes anti-oecuméniques, anti-catholiques et anti-communistes. Le réseau s'est constitué face à l'institution dont il dénonce l'ingérence: le Conseil Oecuménique des Eglises en Amérique latine. Les individus s'organisent en réseau face aux institutions. Ces pasteurs se sont mobilisés face à ce qu'ils dénoncent comme une dérive séculière des protestants. En revendiquant la défense des opprimés dans l'espace du temporel, les protestants auraient négligé le devoir d'évangélisation et la dimension proprement religieuse des Eglises réformées. La régionalisation de CONELA est une réponse face à la domination intellectuelle des théologiens protestants. Au delà de la critique du COE et de son oecuménisme, c'est l'Eglise catholique qui est visée. Le réseau des individus, en l'occurrence les pasteurs des Eglises évangéliques les plus importantes du sous-continent comme les grandes Eglises pentecôtistes chiliennes, combat les institutions et revendique le droit à la représentation religieuse en Amérique latine. Le réseau des individus consacre une forme d'organisation où chacun des membres conserve son indépendance. Les téléévangélistes apportent une caution morale et financière à ce système qui prétend supplanter le protestantisme traditionnel en Amérique latine et combattre l'Eglise catholique. D'un point de vue politique, au Chili comme au Guatemala par exemple, les représentants de ces associations, qui forment les groupes majoritaires, se sont mobilisés contre l'Eglise et se sont rapprochés de l'Etat afin de légitimer son action alors que l'Eglise catholique, par l'intermédiaire de certains de ses évêques ainsi que de bon nombre d'Eglises protestantes, semblaient occuper une place dans l'opposition aux régimes politiques en place. Ces mobilisations contre l'Eglise et en faveur de l'Etat ne sauraient être envisagées comme des phénomènes isolés les uns des autres, des concertations entre les différents dirigeants ont eu lieu afin de définir une ligne de conduite politique, ainsi que la théologie de nature à légitimer cette conduite. Ainsi, la dynamique diplomatique a favorisé une mobilisation transnationale contre les institutions catholique ou protestante de la part d'Eglises qui souhaitent balayer l'identité protestante en Amérique latine en édifiant un monopole évangéliste.

6 Les enjeux du politique : crises, stratégies et transnationalité Le développement des Eglises évangéliques permet de s'interroger sur les structures du pouvoir au sein de ces espaces de diffusion. Le pouvoir se déplace à partir des sphères traditionnelles et légales. Ce déplacement est concomitant à la création de légitimités sociales et à l'émergence de nouvelles allégeances. Les évangélistes se déplacent dans un champ social fragmenté, marqué par la prégnance de la crise: crise sociale, économique, politique et identitaire. Cependant certains points communs doivent aussi être dégagés, à l'image du référentiel face auquel ces groupes ne manquent pas, implicitement ou explicitement, de se définir: l'Eglise catholique. Comment rendre raison de la formation sociale de ces groupes évangélistes? Il semblerait que la dimension de la crise déjà évoquée constitue un des thèmes centraux autour duquel pourrait s'articuler leur émergence. La crise revêt en Amérique latine plusieurs aspects. Une crise de l'Etat 
qui relève d'un défaut de ses prestations, et une crise des valeurs religieuses et des pratiques spécifiquement catholiques ${ }^{21}$. Ces deux dimensions ne sont pas isolées l'une par rapport à l'autre, bien au contraire, des interactions entre ces deux phénomènes seraient lisibles. Il nous faudrait ici tenir compte d'une des données historiques, sociales, politiques et religieuses de tout premier ordre au cours de ces dernières années : l'avènement de la théologie de la libération, puis sa remise en cause par le Vatican. Ce mouvement issu des hiérarchies catholiques latino-américaines les plus progressistes s'était donné comme cadre théologique et comme objectif une synthèse entre la pensée religieuse du catholicisme et les développements du marxisme ${ }^{22}$. La théologie de la libération a connu ses heures de gloire pendant les années soixante-dix en permettant à certains religieux et à certains évêques de dénoncer l'échec social auquel ils étaient quotidiennement confrontés. La sphère du politique ou étatique, caractérisée par la carence de prestations sociales et par le désarroi qui en découle sur le plan collectif, interagit avec la sphère du religieux, au sein de laquelle se développe une mouvance nouvelle qui viendrait, en retour, investir le social. Or, et ce point est particulièrement intéressant lorsque l'on envisage le développement des groupes évangélistes, certains catholiques, proches de la théologie de la libération, s'étaient à de nombreuses occasions prononcés en faveur de l'oecuménisme, permettant à des protestants et à des pentecôtistes de les rejoindre au sein de communautés locales nouvellement constituées, les communautés ecclésiales de base. Les années soixantedix et les années quatre-vingts représentèrent ainsi une période au cours de laquelle la centralité de l'Eglise catholique avait été mise en question en Amérique latine. L'Eglise était alors d'autant plus menacée que s'engageait un processus de dédifférenciation religieuse, dont la logique portait à terme une remise en cause de l'identité catholique en tant que telle dans le sous-continent ${ }^{23}$. Cette menace de décentralisation s'est accompagnée de l'émergence d'une religion populaire spécifiquement latinoaméricaine, le fruit d'une hybridation du christianisme et des cultures locales de ces pays $^{24}$. Dans un tel contexte, la logique de l'essaimage, qui caractérise la dynamique des mouvements évangélistes porte amplement ses fruits, d'autant plus qu'elle se construit sur les bases d'une anomie sociale à la fois urbaine et rurale. Les évangélistes se constituent comme force là où l'Eglise, à travers la logique de l'inculturation, a rencontré ses limites. Ces groupes s'inscrivent à l'intérieur d'un champ urbain dévasté, dans l'espace fragmenté du barrio, ou bien dans les campagnes déstructurées par le chômage et l'exode rural, et essayent de recréer par l'intermédiaire du contrat qui les lie à ses membres une vie à la fois associative et communautaire. Le choix à l'origine de ce contrat encourage les individus à l'action, et définit un groupe motivé par la volonté et la foi. La dynamique sociale des Eglises évangéliques est fidèle à la logique de l'alliance, un contrat à partir duquel les sympathisants se convertissent à ce nouveau culte qui les fera s'intégrer à une vie de quartier, à travers des rites où leur prise de parole sera fortement sollicitée : par exemple le rite de "l'oración» ${ }^{25}$. La thématique millénariste (la fin du monde approche, elle correspond au deuxième millénaire) est propice au fatalisme. L'oración constitue un exutoire pour chaque fidèle, en lui donnant la possibilité de faire état des malheurs qui le frappent. Ce rite soude les liens communautaires et permet l'intégration rapide des nouveaux venus. Le contrat et la logique associative qui le sous-tend participent ainsi à la construction d'une véritable communauté. Le contrat soude le groupe et favorise la consolidation de relations étroites entre ses membres. Ses liens sont d'autant plus forts que la proximité avec le chef est importante. La désignation du chef à l'intérieur des cultes pentecôtistes se fait 
principalement parmi les fidèles. Cette caractéristique de la reproduction de ses dirigeants revêt une importance particulière d'autant plus que la majorité des prêtres catholiques sont, dans nombre de pays d'Amérique latine, d'origine étrangère, occidentaux pour la plupart ${ }^{26}$. La formation des dirigeants des mouvements pentecôtistes ou des autres cultes évangélistes s'est premièrement effectuée grâce à l'apport des pasteurs missionnaires, principalement nord-américains. De ce point de vue, l'analyse d'un groupe nommé Wycliffe Bible Translators - Summer Institute of Linguistics est tout particulièrement significative ${ }^{27}$. Cette institution nord-américaine, une des formations missionnaires les plus importantes au monde par le nombre de pasteurs qui en dépendent ${ }^{28}$, est présente en Amérique latine depuis les années 30 et s'est progressivement développée à l'échelle continentale. Ainsi, de nombreux latinoaméricains, parfois même des indiens, ont été socialisés et formés à ces nouveaux cultes, en tant que simples fidèles ou bien en tant que pasteurs. On a assisté dès lors à une rapide autonomisation locale des membres et des dirigeants des groupes évangélistes, le groupe religieux se voulant autonome et solidement ancré dans les réalités de l'espace social qu'il investit. Les évangélistes rejoignent la culture latinoaméricaine également d'un point de vue symbolique, à travers les relations d'autorité qu'elles instituent entre le chef et les adeptes du groupe. A certaines occasions, on remarque ensuite que la relation entre le chef et ses fidèles s'inscrit en parallèle avec d'autres formes d'autorité, ou de paternalisme, caractéristiques de l'espace culturel latino-américain. Ces représentations du pouvoir sont incarnées notamment par le patron (terme générique pour désigner une figure de l'autorité), l'hacendado (le propriétaire terrien), le cacique (le notable et le protecteur, pilier du clientélisme), ou le caudillo (le chef militaire) ${ }^{29}$. Les Eglises évangéliques sont susceptibles d'organiser des espaces de mobilisation là où les services sociaux de l'Etat font défaut. L'usage de la transnationalité leur permet d'accéder à une reconnaissance dans l'espace public. Au Nicaragua, les années soixante-dix ont vu l'essor d'une frange protestante principalement pentecôtiste. Le tremblement de terre de 1972, puis la révolution sandiniste de 1979, peuvent être considérés comme les tournants majeurs du développement des pentecôtismes dans ce pays. Presque $60 \%$ des évangélistes du Nicaragua se définissent en tant que pentecôtistes. En outre, les effectifs de ces églises auraient augmenté de $90 \%$ entre 1975 et $1979^{30}$. Lors du tremblement de terre, certains évangélistes s'étaient regroupés en un comité d'urgence, le CEPAD (Comitado Evangelico para la Ayuda al Desarollo) afin de recueillir l'aide aux victimes. Dans la lignée du CEPAD, une autre organisation a été formée, la CELADEC (Comision Evangelica de Educacion Cristiana), et a oeuvré à la campagne d'alphabétisation pendant la période révolutionnaire. Cette organisation était affiliée indirectement à la CELA (Confederacion Evangelica latino-americana) qui avait accueilli déjà en 1969, lors de sa troisième conférence à Buenos Aires, 43 Eglises et mouvements protestants. Le versant protestant et oecuménique que la CELA représente ne détenait pas le monopole de la représentation religieuse au Nicaragua. Aussi, l'Eglise du Verbe, une Eglise qui revendique fermement son identité évangéliste et anti-oecuménique, a installé une Eglise à Managua en 1979, dès l'arrivée des sandinistes au pouvoir afin d'apporter de l'aide aux populations en détresse ${ }^{31} \cdot{ }^{32}$. Pour les dirigeants de l'Eglise du Verbe, il s'agissait avant tout de sauver des âmes mises en péril par l'idéologie marxiste des révolutionnaires. Force est de constater qu'indépendamment de leurs options idéologiques, le travail social des évangélistes a été reconnu par les dirigeants révolutionnaires. Aussi, en 1982, Daniel Ortega, le chef des sandinistes, avait tenu à 
souligner « le rôle positif que les églises évangéliques (avaient joué) dans le processus de reconstruction nationale " ${ }^{33}$. Dans l'espace public, le recours à la transnationalité permet à l'Etat d'accéder à une reconnaissance religieuse que l'Eglise catholique a refusé d'accorder à certains régimes. La CONELA, affiliée à la World Evangelical Association des Etats-Unis, a apporté son soutien au gouvernement de Pinochet pendant les années soixante-dix jusqu'à la fin des années quatre-vingts à travers son représentant chilien, ou au régime d'Efraïn Rios Montt au Guatemala au cours des années quatre-vingt (par l'intermédiaire de l'Alianza Evangelica de Guatemala). Alors que le CELA s'était prononcé en faveur de l'oecuménisme, la CONELA s'y était opposée violemment en rejetant par là même très fermement la théologie de la libération ${ }^{34}$. Ces relations transnationales sont organisées en fonction d'une bipolarité issue de la guerre froide. Tout particulièrement depuis le début des années quatre-vingts, deux espaces de mobilisation s'affrontent: les Eglises affiliées au référent institutionnel du Conseil Oecuménique des Eglises de Genève de tendance progressiste et des réseaux conservateurs soutenus par de puissantes logistiques mobilisatrices qui intègrent, entre autres, les ressources des groupes nord-américains. Cette bipolarité formelle ne saurait cependant résister aux stratégies individuelles des Eglises et des groupes ${ }^{35}$ qui recomposent leurs affiliations et leurs allégeances au gré des contextes sociaux.

7 L'action politique des évangélistes en Amérique latine : un essai de typologie Comment caractériser l'action de ces Eglises à l'intérieur des systèmes politiques du souscontinent latino-américain? La mobilité stratégique des acteurs fait partie de nos hypothèses théoriques. Afin de mieux expliciter une situation historique, sociale et politique complexe, nous avons voulu classer en trois catégories les différents pays suivant la nature de leurs régimes politiques. Aussi, nous aborderons les cas du Nicaragua, du Guatemala et du Chili sous l'aspect de l'autoritarisme, (la révolution sandiniste, le gouvernement de Rios Montt, le régime de Pinochet). Le Mexique, la Bolivie et l'Equateur seront appréhendés sous la forme de régimes semi-autoritaires, des systèmes politiques organisés suivant le principe d'élections qui n'excluent pas l'alternance (par exemple en Bolivie et en Equateur en 1981), malgré la présence d'oligarchies puissantes. Enfin, nous envisagerons l'action de groupes évangélistes au sein de systèmes compétitifs dans des pays comme la Colombie ou le Pérou. La relation entre évangélisme et autoritarisme revêt une signification toute particulière. Au Guatemala, au Nicaragua, et au Chili, la place de l'Eglise catholique est une des variables les plus importantes lorsque l'on veut envisager ce problème. En effet, à l'occasion de coups d'Etats ou de révolutions, certains groupes pentecôtistes se seraient constitués en tant que religions légitimes face à l'Eglise catholique qui occupait alors, à plusieurs niveaux hiérarchiques différents, aussi bien à la base qu'à certains sommets, une fonction contestataire. Une véritable concurrence religieuse s'est organisée, et les groupes évangélistes ont participé à des actions politiques, en concourant ainsi pour leur reconnaissance religieuse officielle (par exemple au Nicaragua lors de la campagne d'alphabétisation). Ces contextes de crise sont propices à l'émergence des mouvements pentecôtistes : crise de l'Etat (coup d'Etat, révolution), crise sociale (chômage, exode rural), crise de l'Eglise (minée par la théologie de la libération et la division qui s'était opérée en son sein), et « crise » naturelle (tremblements de terre au Nicaragua en 1972 et au Guatemala en 1976). Comme on peut aisément le remarquer plusieurs dimensions de la crise sont liées les unes aux autres : par exemple la crise de l'Etat et la crise sociale, ou bien les crises naturelles mettant d'autant mieux en lumière la crise sociale et le défaut de protection étatique. Par ailleurs, la fonction manifeste de conformisme 
apolitique que les mouvements pentecôtistes et néo-pentecôtistes semblaient prôner, en arguant que la seule autorité vient de Dieu, et qu'en conséquence l'individu est mis en demeure d'accepter la réalité comme une manifestation de «Sa » volonté, s'était doublée d'une fonction latente de conservatisme. En rejetant le politique comme mode d'action sur le monde et la société, en rejetant la catégorie de l'action si elle n'est pas religieuse et prosélyte, les évangélistes sont amenés, en principe, à cautionner les pouvoirs politiques quels qu'ils soient. Les régimes autoritaires les ont le plus sollicités, et bon nombre de ces mouvements ont répondu à leurs appels ${ }^{36}$. Leur mode de participation à la vie politique se caractériserait alors par une action de type "loyalty " ${ }^{37}$. Aussi, la dimension millénariste fortement présente dans la théologie évangéliste s'est facilement adaptée à un contexte autoritaire. Le chef a fait office de sauveur de l'humanité et l'utopie politique a rejoint l'utopie religieuse. Il est possible de mettre en lumière au Mexique, en Bolivie et en Equateur, l'action d'intermédiation opérée par certains mouvements religieux entre des sous-groupes (minorités ethniques ou sociales) et les gouvernements en place ${ }^{38}$. Des instances évangéliques ont appuyé les revendications de groupes comme les indiens ou les mestizos ${ }^{39}$ face à l'Etat, une action qui tendrait à marginaliser le rôle de l'Eglise, traditionnellement dévouée à la socialisation de ces populations. Nous sommes ici confrontés à une relation de type "voice». Aussi bien au Mexique qu'en Bolivie, pour des raisons différentes, l'Eglise s'est trouvée en difficulté, dégageant un espace de mobilisation pour les mouvements pentecôtistes. L'identité catholique a été mise à mal à l'intérieur de ces pays, notamment du fait de la résurgence des coutumes locales et des rites populaires. Les évangélistes s'accommodent fort bien des cultures locales des lieux où ils s'implantent, et sont ainsi en mesure de concurrencer facilement l'Eglise sur ce terrain. Le retrait de l'Eglise a favorisé l'émergence des Eglises évangéliques, dans des espaces sociaux qu'il est possible d'identifier, là où cette corrélation repose sur des faits ${ }^{40}$. Un désencadrement de la prêtrise et une forte proportion de prêtres étrangers favoriseraient l'émergence des leaders pentecôtistes, proches des lieux de leur action spirituelle, et proches de leurs fidèles par des origines culturelles et sociales communes. La proximité physique, spirituelle et culturelle des chefs religieux vis-à-vis de leurs membres est un des avantages comparatifs majeurs des mouvements évangélistes ${ }^{41}$. Les révoltes indiennes de Bolivie et de l'Equateur en 1990 sont significatives à cet égard de la mobilisation d'une frange de l'Eglise, une partie du clergé local en rupture avec l'ordre du Vatican. Ces mouvements sociaux sont également marqués par la présence d'évangélistes qui n'ont, en ce qui les concerne, d'ordre que celui de leurs groupes autonomes, largement plus mobiles que leurs pairs catholiques. Les systèmes compétitifs sont-ils favorables à la participation des groupes évangélistes ? Des tentatives d'insertion à l'intérieur de ces systèmes politiques ont été remarquées. Au Venezuela, pendant les années soixante-dix, les évangélistes ont soutenu l'initiative de créer un parti. Au Pérou, l'élection de Fujimori en 1990 s'est réalisée avec l'appui de certains groupes pentecôtistes présents dans le pays. Ensuite, de nombreux membres de groupes évangélistes ont fait leur apparition au sein même du gouvernement. Ces deux cas mettent en valeur une situation où des réseaux de solidarité informelle, principalement dans les quartiers populaires des grandes villes, ont oeuvré pour soutenir ou produire des acteurs légitimes de la vie politique du pays. C'est bien du passage du social au légal dont il est ici question, une officialisation que les évangélistes recherchent afin de consolider leur action et leur présence. Ce passage d'un mode à l'autre de l'expression politique et sociale, du fait social au fait légal et 
institutionnel, reste encore à franchir. Une association internationale de partis évangélistes latino-américains a été créée en 1993 aux Etats-Unis, et la première réunion a eu lieu à Caracas au mois d'août de la même année. Cette initiative qui regroupait des formations de nombreux pays latino-américains (Guatemala, Nicaragua, Mexique, Panama, Salvador, Venezuela, Colombie, Pérou, Bolivie) a été structurée avec l'aide de groupes nord-américains présents depuis de nombreuses années dans des pays comme le Guatemala ${ }^{42}$. Ces partis, pour la plupart sans grande expérience politique, sont décidés à partager leurs idées et à se mobiliser contre ce qu'ils considèrent être les fléaux latino-américains : le poids des bureaucraties, l'Eglise catholique, la misère et la corruption des élites. Ainsi, un effort est accompli dans la construction d'une institution transnationale visible et légitime, capable de fédérer sur le terrain de la politique partisane les différentes volontés individuelles. Cette association internationale de partis représente l'aboutissement de nombreuses réunions qui ont eu lieu aux Etats-Unis et en Amérique latine. Elle témoigne également du souci de participer au jeu des élections en démocratie et de s'adapter aux différents contextes politiques issus des vagues de la démocratisation en Amérique latine. La logique du réseau transnational permet l'activation de cette organisation et sa consolidation en dégageant les ressources nécessaires à la formation des futurs dirigeants politiques. Aussi, un des centres de rencontres est une université située en Virginie, l'université du télé-évangéliste Pat Robertson, où des cours de formation sont ouverts pour les dirigeants latino-américains. Indépendamment des succès pour l'instant relatifs de ces formations politiques, il est intéressant d'identifier les lieux de socialisation et de diffusion du savoir de ces nouvelles élites politiques et sociales. Face à l'échec des mobilisations partisanes isolées, depuis une dizaine d'années, une nouvelle mobilisation intègre davantage le recours à la transnationalité et une dynamique centralisatrice. De même, s'inscrit ici en parallèle un autre enjeu, la tendance vers l'élitisme dans le recrutement des mouvements évangélistes. Ce phénomène concerne essentiellement les nouvelles Eglises néo-pentecôtistes. Une partie de la classe moyenne semble désormais touchée, un fait qui viendrait corroborer les hypothèses et les développements des sociologies de Weber et de Troeltsch ${ }^{43}$, alors que certains dirigeants, hommes politiques, hommes d'affaires, ou journalistes, rejoignent les rangs de ces évangélistes. C'est dans ces Eglises que l'intérêt pour la politique partisane et gouvernementale est le plus fort.

8 Les évangélistes dans un monde multi-centré : la construction du rapport à l'autorité La diffusion des évangélistes dans l'espace régional latino-américain nous engage à poser différents types de relation à l'autorité qui se constituent dans une perspective transnationale. La question de l'autorité et du rapport au gouvernement temporel, demeure un des enjeux fondamentaux dans les débats qui animent les dirigeants évangélistes en Amérique latine. L'absence d'autorité verticale et centrale implique un pluralisme dans la définition du rapport au politique et du comportement à adopter face au pouvoir temporel. Le monde multi-centré est l'arène dans laquelle évoluent les évangélistes et le cadre contextuel à l'intérieur duquel il faut situer leur action. L'analyse du discours des acteurs et de leurs mobilisations dans cet espace politique permet d'identifier des rationalités religieuses et sociales confrontées aux logiques des Etats. Les évangélistes occupent une place intermédiaire: entre le repli quiétiste, consacré par la sortie de l'allégeance citoyenne, et la participation dans le système politique traditionnel qu'une nouvelle génération de dirigeants préconise. Ce conflit exprime des tensions entre les principaux dirigeants et leurs identifications dans les 
différents espaces transnationaux. L'absence de centre favorise la multiplication des acteurs religieux. Sans d'autre référent que la parole prophétique, les évangélistes expriment une pluralité de positions autour d'un texte en perpétuelle relecture. En Amérique latine, plusieurs courants semblent se dégager : depuis le refus de l'Etat, de l'Eglise catholique et de la laïcité, jusqu'à la soumission au gouvernement temporel, les variantes sont nombreuses. Ces choix témoignent de l'interaction entre les rencontres mondiales des dirigeants et les conditions politiques internes des pays à l'intérieur desquels ils évoluent. Ces positions dans les sociétés internes sont susceptibles d'être analysées depuis le point de vue de la transnationalité. Ainsi, Susan Strange annonce ce renversement de perspective dans les sciences sociales en engageant le politologue à penser la politique interne à partir des acquis de l'économie politique internationale ${ }^{44}$. Dans l'espace religieux, l'observation des différentes logiques transnationales éclaire les différents rapports à l'autorité des mouvements évangélistes en Amérique latine. Quatre modèles semblent se dégager. Certaines Eglises, à l'image du mouvement théocratique, une Eglise chilienne dont le dirigeant a été ordonné évêque aux EtatsUnis au début des années 1980, ont pendant longtemps prôné le refus de la soumission au gouvernement temporel. Le projet fondamentaliste, tel qu'il s'énonce dans leur mobilisation, témoigne d'une interaction entre des foyers de violence contestataire aux Etats-Unis et des groupes en Amérique latine dont le référent supranational donne un sens aux mobilisations. Ce type de mouvement social exprime une contestation radicale mettant en cause la notion même de système international défini en tant que système interétatique. Le pasteur de l'Eglise occupe une fonction de guide à la fois théologique et politique, investi en tant qu'évêque par une autorité supranationale. A travers la transnationalité, la logique de l'investiture donne lieu à une logique de la contestation de l'autorité des Etats. Ce type de mobilisation ne représente en aucun cas l'expression du courant majoritaire des Eglises évangéliques en Amérique latine. Il concerne certaines Eglises pentecôtistes et néo-pentecôtistes identifiées le plus souvent en tant qu'Eglises charismatiques. Le messianisme religieux se projette dans l'espace politique provoquant la réaction contestataire radicale et structurant le mouvement social suivant le modèle de l'entreprise et de l'armée. Les trois mobilisations, militaire, entrepreneuriale et religieuse, sont interdépendantes et se rencontrent dans le même messianisme d'une Eglise, résolue à exprimer son désir de changement et à revendiquer son droit à la représentation sociale dans l'espace politique. Bon nombre d'Eglises refusent fermement toute forme de participation dans la vie publique et dans les affaires politiques partisanes et gouvernementales. La sortie de l'allégeance est déclarée en tant que telle, et revendiquée au nom d'une citoyenneté du royaume de Dieu à laquelle le croyant se doit d'accéder. Le messianisme religieux obéit ici à une rigoureuse logique fondamentaliste : le pouvoir et l'autorité sont les attributs de Dieu et les dirigeants du gouvernement temporel sont soumis à la volonté divine. Dès lors, il serait vain de se révolter ou de porter un jugement sur un univers préétabli dont la construction échappe à la volonté de l'homme. A travers les rencontres transnationales qui s'organisent entre les différents dirigeants, c'est la même indifférence à l'égard de l'ordre temporel qui s'affiche. Des Eglises regroupant un nombre important de fidèles, comme l'Eglise évangélique pentecôtiste du Chili ${ }^{45}$, sont concernées par cette théologie. Dans son fondement, cette théologie conteste radicalement le pouvoir de l'Etat en statuant définitivement sur le défaut de légitimité qui le frappe: la légitimité des gouvernants est relative, la loi de Dieu est supérieure à la loi des hommes. Cependant dans bon nombre de pays d'Amérique latine, comme au Guatemala, au Nicaragua, ou au 
Chili, ces Eglises sont toujours bien accueillies par les gouvernements à cause de leur discours piétiste, de leur théologie de la « résignation » réductrice des tensions sociales. La transnationalité permet l'expression de discours de mobilisation qui témoignent du désir d'affirmer la responsabilité des évangélistes dans la direction des affaires politiques des Etats. Les Eglises néo-pentecôtistes sont tout particulièrement concernées par ce nouveau messianisme religieux. La mobilisation transnationale stimule la volonté participative des acteurs religieux dans les systèmes politiques internes. En outre, les relations avec des groupes nord-américains qui évoluent dans l'orbite de la nouvelle droite religieuse est un élément précieux dans la compréhension et l'analyse de ces phénomènes en Amérique latine. Ce désir de participation s'est concrétisé au Guatemala à deux reprises, lorsque deux évangélistes ont eu accès à dix années d'intervalle en 1982 et en 1991 à la présidence de la République. Des réseaux de formation des dirigeants s'organisent autour de ce projet hiérocratique, dans un univers religieux qui recherche des signes de l'élection divine dans la participation des chrétiens à la vie politique. La logique politique des évangélistes consiste à ouvrir des espaces de mobilisation pour les Eglises, comme en témoigne le recours aux Eglises lors des présidences évangélistes au Guatemala ${ }^{46}$. La transnationalité dans la mobilisation politique conduit à une réactivation de la transnationalité en augmentant l'espace d'intervention de l'Eglise et de ses dirigeants. Une logique de concertation transnationale s'est exprimée tout particulièrement au cours des années 80 . Un réseau régional de pasteurs, la CONELA, s'est formé avec notamment pour objectif de penser les relations avec les gouvernants. Dans les sociétés internes, cette concertation a donné lieu à une soumission des Eglises au pouvoir des princes. La soumission aux gouvernants obéit à une logique qui désigne le prince comme le représentant de Dieu. En rendant hommage aux dirigeants de l'Etat, les pasteurs reconnaissent le pouvoir de Dieu. L'autorité n'émane donc pas des souverains du temporel : l'autorité est le propre de la souveraineté divine. Dans les discours et la théologie évangéliste l'indépendance des acteurs religieux est ainsi préservée (Dieu est le seul tenant de la souveraineté), tandis que l'inscription dans le registre populiste de l'Etat est rendue possible, comme en témoigne le soutien des Eglises aux régimes militaires de Rios Montt au Guatemala en 1982 et de Pinochet au Chili. Cette soumission à la logique de l'Etat masque la contestation d'une autre institution, structurée dans les relations internationales également en Etat, l'Eglise catholique. Cette transnationalité qui rassemble bon nombre de dirigeants pentecôtistes, parfois certains baptistes ou des presbytériens indépendants, est le fruit d'une stratégie de contestation de la relation privilégiée qui existe dans bon nombre de pays d'Amérique latine entre l'Etat et l'Eglise catholique. Du point de vue des relations internationales, cette mobilisation vise à mettre en cause un élément du système interétatique : le monopole de la représentation religieuse dans l'espace institutionnel de l'univers des Etats que l'Eglise catholique a su préserver dans de nombreux pays d'Amérique latine. Ces différentes expressions transnationales témoignent de la capacité des acteurs de relire leur tradition, en fonction d'un contexte et des ressources de l'espace socio-politique à l'intérieur duquel ils s'inscrivent. La connaissance de leurs stratégies au sein de l'espace continental semble éclairer la compréhension de leur action dans les différentes sociétés internes. L'étude de ces mouvements à l'échelle de plusieurs pays permet de dégager une économie politique transnationale structurée en flux et en réseaux. Cette perspective d'analyse tient compte de l'individuation des rapports sociaux propres à cet univers de relations internationales. Des mobilisations identitaires s'organisent sur la base d'une 
fragmentation des allégeances et de la multiplication d'acteurs sociaux en prise sur la transnationalité et la mondialisation des échanges. L'identification à un sur-moi collectif correspond dès lors à une pratique en déclin, comme le soulignent à la fois Norbert Elias et Jürgen Habermas. Les évangélistes opèrent dans ce sens en multipliant les espaces de référence qui rendent caduc le monopole des allégeances en faveur des institutions. Ces mouvements identitaires dans les relations internationales témoignent de la pluralité des référents que les individus mobilisent dans leur rapport au politique et qui participent à la définition de leur rapport à l'autorité. L'autorité correspond dès lors à une dimension dont il faut souligner la nature diffuse et fragmentaire. En prise sur les réalités du monde contemporain, les évangélistes oeuvrent à une multiplication des espaces d'autorité à travers la transnationalité, soucieux d'étendre le champ concurrentiel de la légitimité. Des réseaux d'entrepreneurs se constituent ainsi. Ces entrepreneurs à la recherche de légitimité sociale investissent des espaces régionaux sur lesquels se projettent ces mouvements identitaires.

\section{NOTES}

1. L'Encyclopedia Universalis définit les groupes pentecôtistes comme des "groupes religieux, généralement du type de la secte, qui placent au premier rang de leur croyance la conviction que toute vie chrétienne comprend nécessairement une réactualisation des charismes de la primitive Eglise" (vol. 17, p. 831). Cette définition intègre le terme de secte que nous éviterons cependant d'employer (voir p.12). 2. Le méthodisme est un courant issu du calvinisme et de la contestation de certains de ses principes. Il a été fondé en 1703 par John Wesley au presbytère d'Epinorth en Angleterre.

3. L'évangélisme est le terme générique utilisé pour identifier le protestantisme qui se développe principalement aux Etats-Unis et en Amérique latine à travers son courant fondamentaliste et pentecôtiste : on parlera d'evangelicos en Amérique latine. L'accent est mis tout particulièrement sur deux caractéristiques : le revivalisme dont ce mouvement se fait l'écho, et son orthodoxie (défense des "vérités" chrétiennes à partir du seul texte biblique). On distingue ici l'évangélisme du protestantisme historique et libéral. Pour cette distinction, on peut se référer aux travaux de Jean-Pierre Bastian. Dans une perspective socio-historique, cet auteur propose des éléments de réponse en soulignant la distinction de poser une barrière entre ces deux courants. Jean-Pierre Bastian, Le protestantisme en Amérique latine, Genève, Labor et Fidès, 1994, 324 p. 4. Les Adventistes du septième jour ont été fondés par un baptiste, les Mormons par un méthodiste et les Témoins de Jéhovah par un presbytérien influencé par des adventistes. Ces trois cultes sont des religions révélées qui déclarent leur séparation du protestantisme et donc, par là-même, de l'évangélisme.

5. Pour une conceptualisation de cette formulation nouvelle des relations internationales voir J.N. Rosenau, Turbulence in World Politics - a theory of change and continuity, Princeton, Princeton University Press, 1990, 480 p., ainsi que Bertrand Badie 
et Marie-Claude Smouts, Le Retournement du Monde sociologie de la scène internationale, Paris, FNSP, 1992, 249 p.

6. C'est en Amérique centrale, au Guatemala, où la présence des évangélistes est la plus importante et la plus significative. D'après certaines enquêtes, les évangélistes regrouperaient près d'un quart de la population et auraient bénéficié de taux de croissance très élevés au cours des années 70 et 80 . Dans les autres pays d'Amérique latine, la croissance a été soutenue, cependant leur présence numérique est moins importante. Dans un pays comme le Chili les évangélistes représentent environ 15 pour cent de la population globale. Au Brésil, les évangélistes occupent une place tout particulièrement significative dans la société : ce pays compte des Eglises qui regroupent des masses importantes de fidèles et les pasteurs de ces Eglises sont courtisés par les hommes politiques. Nous ne ferons pas référence au cas brésilien qui mériterait à lui seul de faire l'objet d'une étude approfondie. Pour une comparaison à l'échelle latino-américaine, on peut voir l'ouvrage de David Stoll, Is latin america turning protestant? the politics of evangelical growth, Berkeley, University of California Press, 1990, 424 p.

7. Bon nombre de ces évangélistes, désignés souvent par le terme de télé-évangélistes à cause de leur recours massif aux moyens de communication, sont nord-américains ou latino-américains. Cependant cette logique transcende le seul cadre régional : un des personnages les plus importants qui réussit à fédérer un nombre considérable d'Eglises et d'organisations lors de ces croisades d'évangélisation en Amérique latine est coréen. Le Dr. Choh dirige l'Eglise évangélique la plus importante du monde : près de 1.000 .000 de personnes en Corée du Sud suivant ses dirigeants et il existe de nombreuses Eglises filiales en Amérique latine, aux Etats-Unis et en Asie. A ce titre, le Doctor Choh jouit d'un prestige exceptionnel dans un univers marqué par le culte de la performance. 8. A la suite de ce désastre, les évangélistes Luis Palau, un argentin dirigeant une organisation dont le siège se trouve aux Etats-Unis, et Pat Robertson, un des chefs de file de la nouvelle droite religieuse aux Etats-Unis, ont apporté leur soutien pour aider les populations en détresse.

9. L'univers évangéliste est structuré autour d'une hiérocratie. Il y a peu de division des pouvoirs. La reconnaissance religieuse que le pasteur incarne lui confère des pouvoir qu'il va utiliser dans l'espace transnational diplomatique.

10. Dans la mouvance du protestantisme libéral, des Eglises se sont établies en Amérique latine à partir de 1860 .

11. En effet, c'est là où se trouvent les ressources économiques des donateurs sollicités à travers le réseau média. Par ailleurs, le système fiscal nord-américain favorise la collecte et l'emploi de ces dons : les dons sont déductibles pour le donateur et l'entreprise qui les collecte, si elle remplit certains critères, est exempte d'impôt. 12. Luis Palau était le cadre d'Overseas Crusade, une organisation missionnaire nordaméricaine.

13. Des organisations comme la Christian Broadcast Network, dirigée par Pat Robertson, sont à l'origine de collectes de fonds pour aider des groupes religieux dans des pays comme le Guatemala ou le Nicaragua et soutenir les dirigeants locaux. Ce type d'initiative est aussi impulsé par les dirigeants latino-américains eux-mêmes, puisque des collectes sont organisées en Amérique latine en suivant les mêmes principes : présenter un projet, puis faire appel à la bonne volonté des croyants. Aussi, des évangélistes guatémaltèques ou chiliens sont désireux de soutenir des missions 
d'implantation à Cuba en sollicitant leurs fidèles à financer ces initiatives missionnaires.

14. Les idées et les principes d'organisation de ces réunions sont calqués sur un modèle qui valorise l'expérience transnationale en lui donnant le statut d'expérience fondatrice. Par exemple au Chili en 1983, l'Eglise des théocratiques avait organisé une réunion en reproduisant le spectacle filmé de la convention d'Amsterdam organisée par Billy Graham, le célèbre télé-évangéliste nord-américain. Les débats s'étaient ensuite déroulés sur la base de cette retransmission télévisée.

15. Ces groupes religieux forment une mouvance souvent désignée par le terme de nouvelle droite religieuse (new christian right) qui inclut des dirigeants comme Jerry Falwell, fondateur de la majorité morale (moral majority). D'autres personnages, comme Pat Robertson, sont désireux de fonder une action politique sur la base d'une théologie politique fondamentaliste. Il existe une importante bibliographie sur ces mouvements. On pourra consulter entre autres Jeffrey K. Hadden et Shupe, Televangelism power and politics on god's frontier, Henry Holt and Company, 1988, 325 p. ; ou Steve Bruce, "Modernity and fundamentalism : the new christian right in America", British Journal of Sociology, vol. 41, n 4, décembre 1990, p. 477-496. 16. L'étude des pentecôtismes chiliens menée par le sociologue Christian Lalive d'Epinay a mis en lumière cette dimension quiétiste du pentecôtisme traditionnel des années 60. Lalive d'Epinay, Christian, El refugio de las masas, Santiago de Chile, Editorial del Pacifico, 1968, $295 \mathrm{p}$.

17. A l'image des Eglises Verbo ou Shaddaï qui se projettent à partir du Guatemala dans d'autres pays d'Amérique latine, des flux transnationaux de missionnaires, d'hommes d'affaires et d'hommes politiques se forment à l'intérieur du sous-continent américain. 18. Les évangélistes s'identifient au peuple d'Israèl tel qu'il est décrit dans la Bible. En conséquence, leur théologie politique est fondée sur l'alliance (covenant) qui unit les hébreux à Dieu et sur la promesse d'élection en échange de l'adoration de ce seul Dieu détenteur du monopole des allégeances. Aussi, cette théologie exprime une pensée politique pré-étatique et permet de mieux comprendre la vocation transnationale des missionnaires ainsi que leur méthodologie. Leur vision du monde se projette sur la vie des groupes, des ethnies et des tribus que les évangélistes sont dans l'obligation de convertir afin de constituer une unité nouvelle suivant les termes de l'alliance. 19. Cette logique a fait l'objet d'une analyse désormais classique par le sociologue allemand Ernst Troeltsch. On peut voir notamment Ernst Troeltsch, "Christianisme et Société, conclusions des Soziallehren par Ernst Troeltsch", Archives de Sociologie des Religions, 11/1961, p. 17. Elle correspond à une volonté de légitimation des acteurs qui correspond dans la pratique à une institutionnalisation progressive marquée par l'inachèvement : processus de régionalisation, formation de dirigeants, organisation de colloques et de forums.

20. Cette terminologie semble plus satisfaisante et élimine la connotation péjorative que le terme de secte implique. Elle répond également à certains critères des sociologues des religions qui se penchent sur l'analyse des nouveaux mouvements religieux. Paris, Colloque de l'Association Internationale de Sociologie, table ronde sur les mouvements religieux, juin 1993.

21. Par exemple au Chili, on peut remarquer et essayer de rendre compte de la montée en puissance d'une religiosité populaire, face à la baisse des pratiques de la religiosité catholique traditionnelle. Voir notamment Sergio Spoerer, op. cit., p. 105. 
22. A ce propos on peut voir un numéro spécial de la revue Archives des Sciences Sociales des Religions, 1990, 71, juillet-septembre, entièrement consacré à cette question, et notamment l'article de Daniel Levine, "L'impact de la théologie de la libération", p. 43-62.

23. Ce phénomène trouve une certaine résonance dans les débats qui ont traversé l'Eglise catholique à propos de l'inculturation.

24. Pour un point de vue sur le catholicisme typiquement latino-américain voir Charles Antoine, "La Religion Populaire en Amérique latine" in Histoire vécue du Peuple Chrétien, Jean Delumeau (dir.), Toulouse, Ed. Privat, tome II, p.331-334, 1979.

25. Capsoli Wilfredo, "La Pedagogia del Protestante", in Peruanistas Contemporaneos, W. Capsoli (dir.), Lima, Concytec, 1986, p. 152-158.

26. Par exemple en Bolivie, les prêtres européens et nord-américains représentaient au début des années 80 près des deux tiers des effectifs nationaux. Sur ce point et pour une analyse de la présence de l'Eglise catholique en Amérique andine, on peut voir l'article de Marie-Danielle Demelas et de Y. Saint-Geours, "L'Eglise catholique dans les Andes : évolution de 1948 à 1984", Problèmes d'Amérique latine, 3ème trim., 1986, p. 65-93. 27. On pourra consulter Is God an American? An anthropological perspective on the Mission of the Summer Institute of Linguistics. ouvrage collectif, IWGIA and Survival International, Copenhagen et Londres 1981, 192 p.

28. Idem.

29. Lalive d'Epinay, Christian "Dépendance Sociale et Religion - pasteurs et protestantismes latino-américains", Archives des Sciences Sociales des Religions, juillet-septembre 1981, 52/1, p. 91.

30. Jean-Pierre Bastian, "Religion popular protestante y comportamento politico en America Central : Clientela religiosa y estado patron en Guatemala y Nicaragua", Cristianismo y Sociedad, $n^{\circ} 88,1986$, p.46.

31. Dans son opération au Nicaragua, cette Eglise guatémaltèque a bénéficié du soutien des missionnaires de Gospel Outreach, le groupe californien qui a fondé Verbo au Guatemala.

32. Mensaje del Commandante Daniel Ortega Saavedra a los pastores evangelicos de Nicaragua, sepembre 1982, Managua, CEPAD, 1982, 15 p., cité in Jean-Pierre Bastian, art. cit., p. 55.

33. Mensaje del Commandante Daniel Ortega Saavedra a los pastores evangelicos de Nicaragua, sepembre 1982, Managua, CEPAD, 1982, 15 p., cité in Jean-Pierre Bastian, art. cit., p. 55.

34. Jean-Pierre Bastian a analysé la formation de groupes et de mouvances sectaires au sein d'institutions nationales ou transnationales dans son article "Protestantismos latino-americanos entre la resistencia y la sumision. 1961-1983", Cristianismo y Sociedad, $\mathrm{n}^{\circ} 82,1982$, p. 49-68.

35. On rencontre parfois des Eglises qui appartiennent en même temps à ces deux organisations idéologiquement et structurellement antagonistes.

36. On peut citer à ce propos les paroles du chef fondateur du Wycliffe Bible Translators / Summer Institute of Linguistics William Townsend : "Power, in its simplest form, can be used for you or against you. The best way to keep it from being used against you is to get an official to start using it for you."

37. Albert Hirschman, Exit, Voice and Loyalty : Responses to Decline in Firms, Organizations and States, Cambridge Mass, Harvard University Press, 162 p. 
38. Cette constatation et cette analyse rejoignent les théories de J.N. Rosenau dans sa reformulation des relations internationales, les Etats seraient confrontés à de nouvelles instances de mobilisation qui revendiquent l'expression des sous-groupes.

39. Nous faisons référence plus particulièrement en ce qui concerne le Mexique aux mestizos et aux indiens en ce qui concerne l'Equateur et la Bolivie.

40. Yves Saint-Geours et Marie-Danielle Demelas, "L'Eglise catholique dans les Andes : évolution de 1948 à 1984", Problèmes d'Amérique latine, 3ème trim., 1986, p. 65-93.

41. Notamment en ce qui concerne les indiens, les relations entre les prêtres catholiques et les fidèles sont marquées par la distance. L'Eglise a essayé d'ordonner des prêtres parmi les indiens au Guatemala, mais cette expérience est restée isolée.

42. Le réseau du télé-évangéliste nord-américain Pat Robertson, candidat malheureux à l'investiture du parti républicain en 1988, a été partie prenante dans la formation de cette organisation.

43. Les thèses wébériennes de la prédestination sont confirmées par la nature des mobilisations de certains mouvements. Par ailleurs, le texte de Weber sert même parfois de support dans une perspective militante, afin d'apporter la preuve scientifique de leur supériorité sur les autres cultures, les autres groupes sociaux et l'Eglise catholique. Troeltsch, quant à lui, considère que l'élitisme des groupes sectaires est une des tendances majeures dans le développement de ces mouvements.

44. Susan Strange, "Traîtres, agents doubles ou chevaliers secourables ? les dirigeants des entreprises multinationales" in Michel Girard (dir.), Les individus dans les relations internationales, Paris, Economica, 1994, p. 217-229.

45. Cette Eglise est, par le nombre de ses membres, la deuxième Eglise évangélique d'un pays où deux millions de personnes environ adhèrent à l'évangélisme. Par ailleurs, il existe des Eglises issues de cette même matrice dans les pays voisins comme l'Argentine et la Bolivie, ainsi qu'aux Etats-Unis.

46. On peut voir entre autres sur ce point Jesus Garcia Ruiz, "Un essai de contrôle des consciences dans un contexte de guerre civile : militaires et populations indiennes au Guatemala", in François Chazel (dir.), Action collective et mouvements sociaux, Paris, PUF, 1993, p. 125-142.

\section{INDEX}

Index géographique : Amérique centrale, Amérique du Sud

Mots-clés : religion, réseaux transnationaux, mouvements politiques, Mouvements sociaux 